\title{
Commentary
}

\section{An overview of intra-articular therapy for mucopolysaccharidosis VI}

\author{
Dyane Auclair $^{\mathrm{a}, *}$, David Ketteridge ${ }^{\mathrm{b}}$, Stephanie Oates ${ }^{\mathrm{b}}$, John J. Hopwood $^{\mathrm{a}}$ and Sharon Byers ${ }^{\mathrm{c}}$ \\ a'Lysosomal Diseases Research Unit, SA Pathology (at Women's and Children's Hospital), North Adelaide, SA, \\ Australia \\ ${ }^{\mathrm{b}}$ Metabolic unit, SA Pathology (at Women's and Children's Hospital), North Adelaide, SA, Australia \\ 'Matrix Biology Unit, SA Pathology (at Women's and Children's Hospital), North Adelaide, SA, Australia
}

Accepted for publication 21 January 2010

Keywords: Mucopolysaccharidosis type VI, lysosomal storage diseases, enzyme replacement therapy, intra-articular therapy, degenerative joint diseases, $N$-acetylgalactosamine-4-sulfatase

\section{Introduction}

In Mucopolysaccharidosis VI (MPS VI) degenerative joint diseases (DJD) develop early on during maturation. Since DJD is refractory to intravenous enzyme replacement therapy (IV ERT) due to the avascular nature of the cartilage, an intra-articular therapeutic approach has been investigated to prevent the development of DJD in major joints.

\section{Intra-articular therapy in MPS-VI cats: Dose-interval study}

In a preliminary study using juvenile MPS VI cats as models, we examined lysosomal clearance in cartilage sample and synovium from joints collected 7, 28, 56 or 86 days after a single intra-articular injection (IA INJ) of 10,100 or $500 \mu \mathrm{g}$ of recombinant human $N$ -

*Address for correspondence: Dyane Auclair, Lysosomal Diseases Research Unit, SA Pathology (at Women's and Children's Hospital), 72 King William Road, North Adelaide, SA 5006, Australia. Tel.: +61 88161 6153; Fax: +61 88161 7100; E-mail: dyane.auclair @adelaide.edu. acetylgalactosamine-4-sulfatase (rhASB) [3]. In each cat, the treated joints (right elbow, shoulder, knee) were compared to the contralateral joints treated with buffer only.

The highest dose was the most efficient at clearing storage material inside lysosomes. Seven days after a single injection of $500 \mu \mathrm{g}$ rhASB into the joint, clearance of vacuolation was seen from the superficial layer up to mid-depth. One month after one IA INJ of $500 \mu \mathrm{g}$ of rhASB, a small amount of re-accumulation of lysosomal storage material had occurred within the chondrocytes located at the surface of the cartilage (ie. in the most metabolically active layer), however, cells deeper in the tissue were still free of vacuolation. Two and three months after one IA INJ of $500 \mu \mathrm{g}$ of rhASB, reaccumulation of storage had occurred within lysosomes and all chondrocytes throughout the full thickness of the articular cartilage contained large vacuoles [3].

In order to prevent the development of DJD or slow down progression, an ideal therapy should benefit each component that plays important structural, functional and mechanical roles in the joint (cartilage, synovium, synovial fluid, ligaments, bone). By monitoring histological changes at the synovial layer, we verified that the benefit of IA INJ of rhASB extended beyond car- 
tilage. In the buffer-treated joints of all MPS VI cats, hyperplasia of the synovium was obvious and the synovial cells appeared large and very foamy. One week after a single IA INJ of rhASB (for all doses), the level of storage material within the synovium was considerably reduced and the majority of synovial cells appeared smaller and only mildly foamy. However, hyperplasia of the synovium was still noticeable in some areas. Re-accumulation of storage material within synovial cells was detectable one month after a single injection of rhASB with synovial cells appearing mildly or moderately foamy. The synovium also exhibited variable degree of hyperplasia. Two and three months after a single injection of rhASB, the appearance of the synovium was similar to that observed in the buffertreated joints. No signs of inflammation were detected in any control or treated joint [3]. Storage in cruciate ligaments was also reduced in knee joint treated with IA INJ [3]. Subchondral bone and synovial fluid were not altered by a single IA treatment (data not shown).

\section{Intra-articular therapy in MPS-VI cats: Long-term study}

In a second study [2], IA INJ of rhASB or buffer were administered monthly or every three months (q3m) from 1 to 10 months of age in the joints of seven MPS VI cats. Four of these seven MPS VI cats were also treated with weekly IV ERT from birth to prevent the development of severe gait abnormalities (eg. due to hindlimb paresis or hip subluxation) assuming that improved penetration into the joints will occur if mechanical forces applied on the supporting joints are maintained near normality. For each MPS VI cat, the joint treated with buffer or with $500 \mu \mathrm{g}$ rhASB q3m (left elbow, shoulder, knee) was used as control for the contralateral joint treated with more enzyme $(500 \mu \mathrm{g}$ rhASB monthly or q3m) (right elbow, shoulder, knee). The joints of two normal cats were left untreated [2].

Although a relatively low number of joints were examined and wide variation existed between animals, a trend for an overall improvement in gross appearance of treated MPS VI joints was recorded seven days after the last IA INJ of rhASB (Fig. 1A-C). Overall, a decrease in severity of DJD was observed as the total dose of rhASB received over the nine-month period increased. For instance, more severe DJD lesions were present in joints treated $\mathrm{q} 3 \mathrm{~m}$ (compared with monthly-treated joints) or in joints treated with buffer only (compared with joints treated $\mathrm{q} 3 \mathrm{~m}$ ).
Most rhASB-treated joints exhibited a clear reduction in vacuolation within the chondrocytes located near the articular surface compared with untreated joints (Fig. 1D-F). In an individual cat, the joints that received rhASB every month or q3m exhibited a considerable decrease in cartilage tissue uronic acid content (up to $\sim 60 \%$ ) compared with the contralateral joints that received rhASB less frequently or buffer only [2].

A difference between the IA-treated cartilage of MPS VI cats administered combined therapy and MPS VI cats administered IA INJ only could not be established, because, contrary to the previous high and low incidence of paresis observed in untreated MPS VI cats and MPS VI cats treated with IV ERT form birth, respectively $[1,6]$, one out of four cats treated with IV ERT from birth developed hindlimb paresis while the three cats that did not receive IV ERT did not develop severe gait abnormalities during the study [2].

For all cats that received IA INJ of rhASB, a noticeable improvement in the histological appearance of the synovium was detected irrespective of the degree of severity of DJD exhibited by the joint (Fig. 1G-I). This suggests that an adequate amount of enzyme was taken up by the synovium after IA treatment. Engorgement of the synoviocytes in buffer-treated joints was reduced in one MPS VI cat treated with IV ERT but not in another cat treated similarly. Since the three buffer-treated MPS VI joints showing synovial improvement only exhibited early stages of DJD while the three buffertreated joints of the other IV ERT-treated MPS VI cat exhibited advanced stages of DJD, it was speculated that enough systemic rhASB is able to reach and affect the synovium as long as this tissue remains structurally intact and well-vascularised (which is most likely to occur for as long as the DJD remains at its early stages). On the other hand, as the DJD reaches more advanced pathological stages, the synovial microvasculature and architecture will gradually become more disorganized and will eventually lose its function. As a consequence of the deterioration in synovial microvasculature, less systemic rhASB will be able to reach the synoviocytes and re-establishment of normal synovium conditions will not be achieved using IV ERT alone [2,7].

In a few synovium samples obtained from rhASBtreated joints and buffer-treated joints, we detected some neutrophils or small confined areas of lymphoplasmacytic proliferation adjacent to the synovium. Eosinophils were not seen in any of the synovium samples. No correlation between presence of inflammatory cells in synovium samples, severity of articular cartilage lesions, and joint treatment could be estab- 


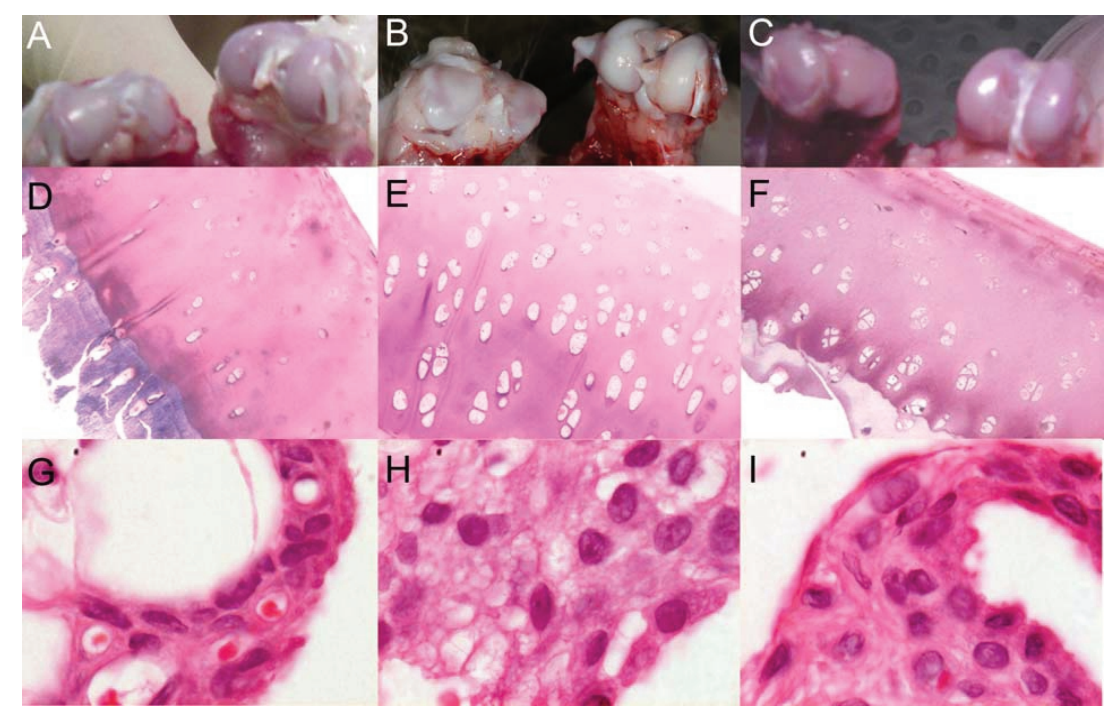

Fig. 1. Joint appearance, chondrocyte and synovial response to IA INJ of rhASB. Gross and microscopic appearance of the articular cartilage, and microscopic appearance of the synovium of an untreated joint obtained from a 10 month-old normal cat (A, D, G), a buffer-treated joint obtained from a 10-month old MPS VI cat (B, E, H) and a joint treated monthly with IA INJ of $500 \mu \mathrm{g}$ rhASB obtained from a 10-month old MPS VI cat (C, F, I). In the normal joint, the cartilage was thin and transparent (A), the chondrocytes were not vacuolated (D) and the synovium usually contained less than two cell layers of unvacuolated synoviocytes (G). In the buffer-treated MPS VI joint, the cartilage was thick and white (B), the chondrocytes were vacuolated due to abnormal accumulation of lysosomal storage (E), and the synovium exhibited signs of hyperplasia (increased numbers of synoviocytes resulting in more than three cell layers) and hypertrophia (enlarged synovial cells containing excessive lysosomal storage conferring a foamy appearance to the cytoplasm). The MPS VI joint treated with monthly IA INJ of $500 \mu \mathrm{g}$ rhASB exhibited thinner cartilage (C), smaller, less vacuolated superficial chondrocytes (F) and smaller, less foamy synovial cells due to reduction in lysosomal storage (I), however, hyperplasia of the synovium was not corrected. Micrographs of cartilage were taken at 200X. Micrographs of synovium were taken at 1000X. More detailed micrographs are presented in ref. [2].

lished [2]. Repeated IA INJ alone did not alter the radiographic appearance of the subchondral bone [2]. Smears of synovial fluid collected from buffer and rhASB-treated joints of MPS VI cats did not display traces of inflammatory exudate [2]. Negligible antirhASB antibody titres were detected at all times in all cats in this study. No adverse events were detected, in particular, the injected joints did not display any outward signs of inflammation in the hours or days following each IA INJ [2].

\section{Intra-articular therapy in MPS-VI human patients: Feasibility clinical trial}

Following such encouraging results using IA INJ of rhASB in MPS VI cats, a small scale pilot clinical trial was undertaken undertaken in Australia (Metabolic clinic, SA Pathology, Women's and Children's Hospital, North Adelaide, South Australia). Two MPS VI patients (16 and 22 years old) receiving weekly intravenous rhASB at the recommended dose of $1 \mathrm{mg} / \mathrm{kg}$ since 2002 were administered $1 \mathrm{mg}$ of rhASB into each hip every two months for one year. Each IA INJ was done under ultrasound guidance after topical application of anesthetic on the skin and local injection of lignocaine. Two MPS VI siblings (five and eight years old) receiving weekly IV ERT of rhASB only were used as controls for this trial. In order to monitor progression of DJD in each patient, baseline and end of trial MRI and ultrasound of both hips were performed. Physiotherapy assessments were also performed at baseline, six and 12 months. A medical review was carried out at the time of each IA INJ. Blood samples were collected at baseline and at the end of the trial to obtain data on anti-rhASB antibody levels, selected immunological parameters (eg. rheumatoid factor), and routine biochemistry.

All IA INJ were well tolerated. Keeping in mind a considerable degree of subjectivity in the comments made by the patients, both patients reported some minor discomfort during the passage of the needle into the joint space during the first IA INJ. Since less discomfort was felt during subsequent IA INJ it was speculated that the joint capsule may have improved as a consequence of the first IA INJ. Both patients described a comfortable "spongy" joint feeling up to six hours after an IA INJ. In some instances, the duration of the 
local anesthesia appeared slightly extended. Overall the main observations (albeit subjective) consisted of improved walking endurance and less joint pain. No adverse events were observed following the IA INJ.

Serial MRI, ultrasound and joint range of motion data are currently being assessed and will be reported upon completion of the analysis. Due to the low number of patients participating in this feasibility clinical trial, extreme care will be taken not to draw conclusions beyond the lack of significant negative events, however, indication of mild improvement of the hip joint or lack of deterioration in contrast to previously observed worsening will be regarded as encouraging results.

\section{Future therapies for joints of MPS VI patients}

Our data demonstrate that rhASB can penetrate the avascular articular cartilage after direct injection into the joints. IA therapy with rhASB could possibly offer a means of slowing or interrupting the progression of DJD in the largest freely movable joints of MPS VI patients treated or not with conventional IV ERT. New intra-articular drug delivery systems (eg. products of nanotechnology) developed to allow sustained delivery of drug in the vicinity of joint, will eventually be used to improve the pharmacokinetic and pharmacodynamic properties of rhASB in order to reduce the need for frequent IA INJ [4]. Meanwhile, the development of other therapeutic options that will offer safe and effective long term correction of the enzymatic defect (eg. gene therapy) needs to be pursued [8]. A gene therapy approach using a lentiviral vector to provide high and sustained $N$-acetylgalactosamine-4-sulfatase levels in the MPS VI joint is currently under investigation [5]. Further studies are needed to evaluate the efficacy and practicality of intra-articular administration of recombinant enzyme to prevent the development of chronic debilitating DJD in MPS VI patients.

\section{Acknowledgements}

The authors gratefully acknowledge Prof. Mark Haskins, University of Pennsylvania for the original heterozygous MPS VI cats and the animal care staff at the Institute of Medical and Veterinary Science for the daily care of the cat colony. We thank Ms. Lyn Waterhouse (Adelaide Microscopy, University of Adelaide) and the Department of Histopathology (SA Pathology, WCH) for tissue preparation and Dr. John W.
Finnie (SA Pathology at Institute of Medical and Veterinary Science, Gilles Plains, South Australia) for regular histopathology assessments. We also thank Dr. Stuart Swiedler and Dr. Charles A. O’Neill, BioMarin Pharmaceuticals Inc. for their regular support and input. We gratefully acknowledge all MPS VI patients and families participating directly or indirectly to the clinical trial. At the Women's and Children's Hospital, we thank the Department of Medical Imaging for MRI and Ultrasound imaging, the Pharmacy Department for enzyme preparation, the Core laboratory for laboratory assessments, Dr. Mike Wilks and colleagues for procedural radiology, Ms Kelly Beard for physiotherapy, and Ms Sophie Lazenkas for administrative support. This work was supported by BioMarin Pharmaceuticals Inc., and the National Health and Medical Research Council of Australia. This manuscript was developed as the result of a meeting of experts entitled "Promoting Bone Health in MPS VI: Framing New Therapies" held in Oakland, California in October, 2008. This meeting was supported by an educational grant from BioMarin Pharmaceutical, Inc., Novato, CA. BioMarin had no role in the content presented and discussed at the meeting.

\section{References}

[1] D. Auclair, J.J. Hopwood, D.A. Brooks, J.F. Lemontt and A.C. Crawley, Replacement therapy in mucopolysaccharidosis type VI: advantages of early onset of therapy, Mol Gen Met $\mathbf{7 8}$ (2003), 163-174.

[2] D. Auclair, J.J. Hopwood, J.F. Lemontt, L. Chen and S. Byers, Long-term intra-articular administration of recombinant human $\mathrm{N}$-acetylgalactosamine-4-sulfatase in feline mucopolysaccharidosis VI, Mol Gen Met 91 (2007), 352-361.

[3] D. Auclair, L.K. Hein, J.J. Hopwood and S. Byers, Intraarticular enzyme administration for joint disease in feline mucopolysaccharidosis VI: enzyme dose and interval, Pediatr Res, 59 (2006), 538-543.

[4] H.M. Burt, A. Tsallas, S. Gilchrist and L.S. Liang, Intraarticular drug delivery systems: Overcoming the shortcoming of joint disease therapy, Expert Opin Drug Deliv 6 (2009), 1726.

[5] S. Byers, M. Rothe, J. Lalic, R. Koldej and D.S. Anson, Lentiviral-mediated correction of MPS VI cells and gene transfer to joint tissues, Mol Genet Metab 97 (2009), 102-108.

[6] A.C. Crawley, K.H. Niedzielski, E.L. Isaac, R.C.A. Davey, S. Byers and J.J. Hopwood, Enzyme replacement therapy from birth in a feline model of mucopolysaccharidosis type VI, J Clin Invest 99 (1997), 651-662.

[7] J.R. Levick, Microvasculature architecture and exchange in synovial joints, Microcirculation 2 (1995), 217-233.

[8] K.P. Ponder and M.E. Haskins, Gene therapy for mucopolysaccharidosis, Expert Opin Biol Ther 7 (2007), 1333-1345. 